Working Paper 49

Business Economic Series 12

October 2008
Departamento de Economía de la Empresa Universidad Carlos III de Madrid Calle Madrid, 126 28903 Getafe (Spain) Fax (34-91) 6249607

\title{
Statistical Properties and Economic Implications of Jump-Diffusion Processes with Shot-Noise Effects*
}

\author{
M. Moreno ${ }^{1}$, P. Serrano ${ }^{2}$ and W. Stute ${ }^{3}$
}

\begin{abstract}
This paper analyzes the Shot-Noise Jump-Diffusion model of Altmann, Schmidt and Stute (2008), which introduces a new situation where the effects of the arrival of rare, shocking information to the financial markets may fade away in the long run. We analyze several economic implications of the model, providing an analytical expression for the process distribution. We also prove that certain specifications of this model can provide negative serial persistence. Additionally, we find that the degree of serial autocorrelation is related to the arrival and magnitude of abnormal information. Finally, a GMM framework is proposed to estimate the model parameters.
\end{abstract}

Keywords: Filtered Poisson Process, Characteristic Function, Generalized Method of Moments JEL Classification: C10, C15, C22, C52, G10

\footnotetext{
${ }^{1}$ University of Castilla La-Mancha, Department of Economic Analysis and Finance, 45071 Toledo, Spain. E-mail: manuel.moreno@uclm.es.

${ }^{2}$ University Carlos III, Department of Business Administration, 28903 Getafe (Madrid), Spain.

E-mail: pedrojose.serrano@uc3m.es.

${ }^{3}$ University of Giessen, Mathematisches Institut, Giessen, Germany.

E-mail: winfried.stute@math.uni-giessen.de
} 


\section{INTRODUCTION}

This paper focuses on a continuous-time process named Shot-Noise JumpDiffusion (SNJD, hereafter) recently proposed by Altman et al. (2008). The SNJD model incorporates the Jump-Diffusion (JD) process introduced by Merton (1976) for modeling stock price movements. According to Merton (1976), the total variation in the asset prices reflects the joint effect of a) the normal changes (owing to the bid-ask crossing of common information and modeled by a Brownian Motion) and b) the abnormal ones (modeled by a Poisson process) originated by the arrival of uncommon information. These abnormal changes in prices produce discontinuities in the stock paths, known as jumps, which may be related to news about corporate defaults, bank crises, etc.

Basically, the SNJD process extends the JD models by adding a term (known as Shot-Noise function) to the Poisson process. As we will see, this additional term tries to reflect some situations that can arise in financial markets such as, for instance, when the effects of a jump fade away in the short/long run. For example, the surprise reaction of the interest rate markets to the auctions of Central Banks is reflected in the presence of jumps in the interest rate quotes. However, the effects of these jumps disappear rapidly, causing the spikes which are present in the interest rate series.

In a similar way, this kind of up-down (down-up) impulses are also present in electricity markets: the spikes are a common shape in spot electricity prices as a consequence of shocks in demand ${ }^{1}$ (Escribano, Peña and Villaplana, 2002). This behaviour is not correctly modeled by traditional JD processes; as will be presented further ahead, the SNJD model fulfills in a simple and elegant manner this trade-off between economic intuition and analytical tractability.

An additional feature of the SNJD model is its ability to introduce serial dependence into financial series. Particularly, we demonstrate that a simple version of the Shot-Noise function produces negative autocorrelation coefficients. Moreover, an additional advantage of the SNJD models is that, as discussed in Bondesson (1988), many statistical distributions appear as marginal distributions for simple Shot-Noise processes.

\subsection{The economic significance of the SNJD model}

We show several different implications of the SNJD model. As was previously mentioned, some economic series exhibit abrupt changes in their value

\footnotetext{
${ }^{1}$ Schmidt (2007) is pioneer in modeling the electricity spikes by using the Shot-Noise process.
} 
in short periods of time. Visually, we can observe how this type of series exhibit a jump followed by a instantaneous (partial) drop back to the previous level (Weron, 2005). These effects are often named spikes in the financial literature $^{2}$. Economically, its origin could be due to changes on the monetary policy (Das (2002) or Benito, León and Nave, 2007) or extreme fluctuations in supply and demand (Barone-Adesi and Gigli (2003) and Lucía and Schwartz, 2002). Financial academics tend to model the spikes by using a mean-reverting process with jumps, where the mean-reversion speed leads the convergence to the long-term value after the jump. As a consequence, estimations of this speed are higher than real. This is erroneous as this speed value is related to the Brownian motion component, and not to the jump one.

As an example, Figure 1 displays the sample path of the European Overnight Index Average (EONIA) rate and its increments. The sample period ranges from $05 / 02 / 2004$ to $23 / 08 / 2005$. This figure illustrates the cited spikes on the EONIA rates. Taking the previous considerations into account, the SNJD model could be considered as an adequate candidate for dealing with such empirical features.

\section{[INSERT FIGURE 1 ABOUT HERE]}

Another possible application of the SNJD model concerns long-range dependence problems. Campbell, Lo, and MacKinlay (1997) refer to longrange memory as observations in the remote past that are nontrivially correlated with observations in the distant future, even as the time span between the two observations increases. As pointed out by Singleton (2006), an important number of studies indicate the presence of some kind of serial dependence in stock returns.

Additionally, Lo (1991) enumerates some possible implications of longrange dependence on financial economics and emphasizes the inconsistency with long-memory of the most common continuous-time models. Moreover, he also concludes that stochastic models for short-range dependence could correctly capture the behavior of asset returns. Our results seem to confirm that a suitable specification of the SNJD model is capable of generating persistence in log-returns, providing support to previous empirical findings.

This type of model can be also interesting for risk management purposes. On the one hand, the estimation of Value at Risk (VaR) and other tail statistics depends dramatically on large price changes. As pointed out by

\footnotetext{
${ }^{2}$ We reserve the name of jumps for those events that, once triggered, return -or not- to their previous position at a lower rate than those for the spikes.
} 
Aït-Sahalia (2004), many of these techniques are oriented to distinguish between eventual jumps and the daily Brownian noise. The presence of a serial dependence pattern in asset returns can lead to a miscalculation in the total volatility of the data generating process, leading to model risk effects.

Finally, the SNJD model can be useful for dealing with EBIT (earnings before interests and taxes)-based models (Lando, 2004) that try to reflect the possibility that, after an announcement of extraordinary profits (jump), the results of the company go back to the previous (pre-jump) level. To the best of our knowledge, we have no evidence (empirical or simulated) about how the persistence through time of jumps can affect the risk management strategy of a firm. We are inclined to believe that the SNJD model could provide additional insights on this issue.

\subsection{Literature Review}

The trade-off between the adequacy of the distribution for capturing the behavior of an economic variable and its analytical tractability currently constitutes an important issue. Nowadays, as pointed out by Duffie, Pan and Singleton (2000), the Affine Jump-Diffusion (AJD) process can provide analytical solutions to many problems in arbitrage-free pricing theory. Moreover, the existence of an econometric theory for its estimation ${ }^{3}$ leads to the current wide range use of ADJ processes in financial economics. For instance, Vasicek (1977) or Cox, Ingersoll and Ross (1985) are some examples of continuous-time based models that model how interest rates evolve through time.

All things considered, do we need to consider a new family of models? This extension is clearly needed if previous models are not able to reflect some empirical features present in economic series. For instance, the Geometric Brownian Motion (GBM) is often presented as paradigmatic: the most popular option pricing model introduced in Black and Scholes (1973) assumes that the price of an asset follows a lognormal distribution. However, the excess kurtosis or volatility clusters detected in financial prices series (see, for instance, Andersen, Benzoni and Lund, 2002) motivate the interest of extending this model.

Additional examples of drawbacks in the current AJD literature can be reviewed also. Regarding interest rate models, Duffee (2002) points out the inner restrictions of standard affine models when it comes to compensating the risk faced by investors ${ }^{4}$, based on the poor performance of AJD

\footnotetext{
${ }^{3}$ See Duffie et al. (2000) or Singleton (2001).

${ }^{4} \mathrm{An}$ interesting discussion about this feature can be found in Singleton (2006).
} 
models when analyzing Treasury yields. On the other hand, Lo (1991) and references therein stress that the continuous-time stochastic processes most commonly employed are inconsistent with the persistence patterns found in asset returns (Campbell et al., 1997), in view of the fact that these models cannot generate serial dependence consistent with long-term memory in returns.

The SNJD model is a further step in the tradition of JD models initiated by Merton (1976) and continued by Ball and Torous $(1983,1985)$ and Jorion (1988). The SNJD model reflects in a simple and intuitive way not just the jump, but also what happens after the occurrence of such jump.

\subsection{Summary of Main Results and Contributions}

This paper provides some additional insights into the SNJD model and contributes to the existing literature in several directions: Firstly, from a theoretical point of view, we provide an analytical expression for the process distribution and several statistical properties, generalizing the results obtained by Das and Sundaram (1999). Secondly, we introduce a brief treatment of the Shot-Noise process in the economic literature. Thirdly, we provide results related to the ability of the model to generate serial persistence in financial asset returns. Additionally, our results imply a close link between the intensity of this persistence and the arrival of the shocking information. Finally, we propose and illustrate the methodology for estimating the parameters of the model. To our knowledge, this is the first study on i) analyzing the statistical applications of the SNJD model in Economics and ii) providing an econometric framework for its estimation.

The structure of this paper is as follows: Section 2 presents a general treatment of the Shot-Noise process and the SNJD model in the statistical literature. Sections 3 and 4 provide the distribution of the SNJD process and its cross moments. Section 5 analyzes the most basic example of the SNJD process. Section 6 carries out the estimation methodology of the process. Finally, Section 7 summarizes the main conclusions.

\section{THE MODEL}

This section introduces the Shot-Noise process and, later, presents the SNJD model and some of its main features. Finally, we compare the SNJD process with the GBM and JD alternatives. 


\subsection{The Shot-Noise process}

Let $H_{t}$ be a stochastic process of the form

$$
H_{t}=\sum_{k=1}^{N_{t}} A_{k} h\left(t-\tau_{k}\right), \quad t \geq 0
$$

where $\left\{A_{k}, k=1, \cdots, N_{t}\right\}$ are i.i.d. random variables, $\left\{N_{t}\right\}_{t \geq 0}$ is an homogeneous Poisson process with intensity $\lambda, h\left(t-\tau_{k}\right)$ represents the reaction to a possible event with magnitude $A_{k}$, and $\left\{\tau_{k}\right\}$ indicates the jump time of the Poisson process. It is also assumed that the variables $A_{k}$ are independent of the Poisson process, $N_{t}$.

Then, $H_{t}$ is named Shot-Noise process and it has been intensively studied by Rice (1954) and Parzen (1962) ${ }^{5}$. Moreover, Bondesson (1988) provides a general treatment of these processes and includes extensive references. For illustrative purposes, Figure 2 represents a simulated path of a Shot-Noise process.

\section{[INSERT FIGURE 2 ABOUT HERE]}

An interesting feature of the Shot-Noise model is that many distributions appear as marginals of this kind (see Bondesson, 1988). This is the reason why the function $h(\cdot)$ plays an important role in the process distribution. As an example, if $\left\{A_{k}\right\}$ is a mixture of exponential distributions and

$$
h(x)=\left\{\begin{array}{cc}
0 & x<0 \\
c \exp (-a x) & x \geq 0
\end{array}\right.
$$

with $c \in \mathbb{R}$ and $a \in \mathbb{R}^{+}$, Bondesson (1988) shows that the Shot-Noise distribution is a generalized gamma convolution.

An additional characteristic of the Shot-Noise process is its ability to introduce autocorrelation in data. Table 1 displays the autocorrelation function $(\mathrm{ACF})$ for the Shot-Noise process with exponential decaying function ${ }^{6}$ $h(\cdot)$. The parameter $a$ controls the decay degree of the process. Each column corresponds to different values of this parameter. For completeness, the last column includes the ACF for an AR(1) model. Different lags are disposed in rows. This Table is directly inspired by Campbell et al. (1997),

\footnotetext{
${ }^{5}$ The Shot-Noise process is sometimes called Filtered Poisson process (Parzen, 1962).

${ }^{6}$ Advancing some results that will be presented later, the ACF of this process is given by $\operatorname{corr}\left[H_{t}, H_{t+n \Delta t}\right]=\exp (-a n \Delta t)$ and can be found in Ross (1996).
} 
where we have employed the Shot-Noise model instead of the fractionally differenced process.

Table 1 reflects that the parameter $a$ modulates the persistence of the process in time: the higher the parameter $a$ is, the higher decay the ACF at first lags is. To put it another way, as a increases, we observe that serial correlation in time diminishes, as it is observed by direct comparison with the $A R(1)$ process column. Generally speaking, we can observe similar patterns of decay between the $A R(1)$ process and the Shot-Noise. Although, as we will see, this autocorrelation pattern is modified in an interesting way when it comes to the SNJD model.

\section{[INSERT TABLE 1 ABOUT HERE]}

\subsection{A model with Shot-Noise effects}

According to Merton (1976), the arrival of abnormal information (for example, news about a company default) can produce a non-marginal change in the price of a certain asset. This change is modeled as a compound Poisson process that reflects the impact of this non-marginal information. Within the current financial literature, these abrupt changes in prices lead to the so-named jumps. As a result of this, Merton (1976) model describes the behavior of stock prices more realistically.

An additional implication of this model is that the effect of a jump on stock prices does not vanish with time. After an upward jump, prices following a JD process do not return to the pre-jump level.

By contrast, it can be thought that these jumps in stock prices fade away in the long run. An intuitive explanation for this decay is that a positive jump is followed by a profit taking, encouraging the investors to sell their assets. Similarly, an abnormal decrease in the asset price can encourage investors to buy such asset. In terms of the stochastic literature, these impulses can produce a Shot-Noise effect.

The Shot-Noise effect is also observable in other markets, such as those of electricity or interest rates. For instance, the spikes, a common shape in electricity prices owing to demand shocks (Escribano et al., 2002), have been analyzed by Schmidt (2007) by using the Shot-Noise process. Additionally, as pointed out by Das (2002) or Benito et al. (2006), the interventions of the Federal Reserve or the European Central Bank can produce jumps. As we will see, the SNJD model can be suitable for reflecting this issue.

Altmann et al. (2008) present a model that captures some of these effects and, thus, can be a more suitable candidate to reflect these empirical 
features than previous models. The SNJD model assumes that the process for the stock price is given by

$$
S_{t}=S_{0} \exp \left[\left(\mu-\frac{\sigma^{2}}{2}\right) t+\sigma B_{t}\right] \prod_{j=1}^{N_{t}}\left[1+U_{j} h\left(t-\tau_{j}\right)\right]
$$

where

- $S_{j}$ denotes the asset price at time $j$

- $\mu, \sigma$ are constants

- $\left\{B_{t}\right\}_{t}$ is a standard Brownian motion

- $\left\{N_{t}\right\}_{t \geq 0}$ is a Poisson process with intensity $\lambda$

- $\left\{U_{j}\right\}_{j}$ is a sequence of i.i.d. jumps

- $h$ is an arbitrary response function

- $\tau_{j}$ are the jump times.

Additionally, $\left\{B_{t}\right\}_{t},\left\{N_{t}\right\}_{t}$, and $\left\{U_{j}\right\}_{j}$ are assumed to be mutually independent.

To our knowledge, the functional form of $h(t)$ has not been reported yet in the financial literature. Thus, for analytical and intuition purposes, we will assume an exponential decreasing function of the form

$$
h(t)=\left\{\begin{array}{cc}
0, & t<0 \\
\exp (-a t), & t \geq 0
\end{array}\right.
$$

where $a \in \mathbb{R}^{+}$is the speed at which jumps decay.

The main advantage of this specification is that we can relate, in a simple form, the results obtained for our SNJD model with the two standard models for stock prices: firstly, the GBM and the JD processes. Both models are special cases of the SNJD process ${ }^{7}$.

Figure 3 displays the simulation of the SNJD process in equation (2) with exponential response function as given in (3). For simplicity, all the simulations will be carried out with jump sizes $U=\exp \left(-\beta^{2} / 2+\beta \epsilon\right)-1, \varepsilon \sim$ $N(0,1)$. This implies $E[U]=0$. The assumption of a lognormal distribution

\footnotetext{
${ }^{7}$ According to the proposed response function, if parameter $a$ is zero, the SNJD model leads to the JD model of Merton (1976). On the other hand, the GBM is obtained in absence of jumps.
} 
for jump sizes is common in the JD literature (see, for instance, Merton, $1976)^{8}$. Moreover, it is convenient as it decreases the number of parameters under estimation ${ }^{9}$.

\section{[INSERT FIGURE 3 ABOUT HERE]}

To illustrate the differences between GBM, JD and SNJD processes, we carried out different simulations of these models. The results obtained are displayed in Table 2. This Table reports, in columns, the mean, some percentiles and the first three autocorrelation coefficients of log-returns. To control for the degree of serial dependence, the SNJD model has been simulated considering $a=0.5,1.0$ and 10.0. Finally, rows are grouped in three different blocks, attending to their frequency -daily, weekly or monthly-.

\section{[INSERT TABLE 2 ABOUT HERE]}

To compare the jump and Shot-Noise effects against the Brownian noise, the simulations in Table 2 have been carried out by keeping the total variance of the process constant. Two main conclusions arise from this Table: Firstly, the SNJD model presents negative autocorrelation coefficients; attending to the value of the parameter $a$, the magnitude of these coefficients differs. Secondly, the existence of serial correlation results in fatter tails: this effect is observable as the frequency declines. Thus, it seems that the presence of serial correlation affects the distribution of the simulated data.

\section{THE STATISTICAL DISTRIBUTION OF THE PROCESS}

This section focuses on the characteristic function of the log-returns of the SNJD process, as a necessary step to analyze the empirical performance of the model with Shot-Noise effects. We also provide expressions for the first moments, and compare our results with those obtained for the JD processes in Das and Sundaram (1999) and Aït-Sahalia (2004).

\footnotetext{
${ }^{8}$ Some studies propose exponential distribution for the jump size. See, for instance, Duffie and Gârleanu (2001) in the context of credit risk markets, or Barone-Adesi and Giggli (2002) and Villaplana (2003) for electricity markets, among others.

${ }^{9}$ Navas (2003) and references therein offers an interesting discussion about this assumption in the JD process literature.
} 


\subsection{Characteristic function}

Let $\left(\Omega, \mathcal{F}_{t}, P\right)$ be a probability space and denote with $0 \leq t \leq T$ the set of trading dates. $\Delta t$ denotes the length of time between two price fixings. The log-return of the stock price between times $t$ and $t+\Delta t$ is defined as $Z_{t}=\ln \left(S_{t+\Delta t} / S_{t}\right)$. Using (2), $Z_{t}$ is given by the sum of three independent random variables, $X_{t}, Y_{t}$, and $H_{t}$, where

$$
\begin{aligned}
X_{t} & =\left(\mu-\frac{\sigma^{2}}{2}\right) \Delta t+\sigma\left(B_{t+\Delta t}-B_{t}\right) \\
Y_{t} & =\sum_{j=N_{t}+1}^{N_{t+\Delta t}} \ln \left[1+U_{j} h\left(t+\Delta t-\tau_{j}\right)\right] \\
H_{t} & =\sum_{j=1}^{N_{t}} \ln \left[\frac{1+U_{j} h\left(t+\Delta t-\tau_{j}\right)}{1+U_{j} h\left(t-\tau_{j}\right)}\right]
\end{aligned}
$$

The term $X_{t}$ pertains to the log-returns of a GBM process. Owing to the independence property of Brownian increments, log-returns generated by these terms alone over a time grid are not serially correlated.

The term $Y_{t}$ reflects the local contribution of jump events on SNJD logreturns in the time interval $(t, t+\Delta t]$. It equals zero if no jump happens. Also, the $Y_{t}$ 's are independent over disjoint time intervals, as follows easily from the independence of the $U$ 's and basic properties of the Poisson process.

If we compute the log-returns of a JD process, like Merton (1976), this just leads to the expressions obtained for $X_{t}$ and $Y_{t}$. Not surprisingly, an additional term, $H_{t}$, appears when it comes to calculate the log-returns in a SNJD process. It is clear that $H_{t}$ has cumulative rather than local character, as it takes all Shot-Noise effects in the past properly weighted into account. Consequently, this part will be in charge of dependencies among the logreturns over time. Actually, as will be pointed out in the next section, this term is the source of the autocorrelation in the SNJD process.

The probability law of the stochastic process $Z_{t}$ can be expressed in terms of its characteristic function: $\Phi_{Z}(\xi)=E\left[\exp \left(i \xi Z_{t}\right)\right]$. The following Proposition presents an explicit expression of $\Phi_{Z}$. 
Proposition 1 The characteristic function of the variable $Z_{t}$ is given by

$$
\begin{aligned}
\Phi_{Z_{t}}(\xi)= & \exp \left(i\left(\mu-\frac{\sigma^{2}}{2}\right) \Delta t \xi-\frac{1}{2} \sigma^{2} \Delta t \xi^{2}\right) \\
& \times \exp \left(\lambda \int_{t}^{t+\Delta t} E[\exp (i \xi \ln [1+U h(t+\Delta t-\tau)])-1] d \tau\right) \\
& \times \exp \left(\lambda \int_{0}^{t} E\left[\exp \left(i \xi \ln \left[\frac{1+U h(t+\Delta t-\tau)}{1+U h(t-\tau)}\right]\right)-1\right] d \tau\right)
\end{aligned}
$$

Proof. See the Appendix

This result includes the one for JD processes without Shot-Noise effects as obtained by Das and Sundaram (1999). Indeed, if we set $h \equiv 1,(7)$ leads to

Example 1 In the case of a JD process, expression (7) reduces to

$\Phi_{Z_{t}}(\xi)=\exp \left(i\left(\mu-\frac{\sigma^{2}}{2}\right) \Delta t \xi-\frac{1}{2} \sigma^{2} \Delta t \xi^{2}+\lambda E[\exp (i \xi \ln [1+U])-1] \Delta t\right)$

\subsection{Moments of the process}

From the characteristic function of the log-returns we may readily derive the pertaining moments.

Lemma 1 Under (2), we have

$$
\begin{aligned}
E\left[Z_{t}\right] & =\left(\mu-\frac{\sigma^{2}}{2}\right) \Delta t+C+D \\
\operatorname{Var}\left[Z_{t}\right] & =\sigma^{2} \Delta t+C^{2}+D^{2} \\
\text { Skewness } & =\frac{C^{3}+D^{3}}{\left(\operatorname{Var}\left[Z_{t}\right]\right)^{3 / 2}} \\
\text { Kurtosis } & =3+\frac{C^{4}+D^{4}}{\left(\operatorname{Var}\left[Z_{t}\right]\right)^{2}}
\end{aligned}
$$


with

$$
\begin{aligned}
C^{n} & =\lambda \int_{t}^{t+\Delta t} E\left[\{\ln (1+U h(t+\Delta t-\tau))\}^{n}\right] d \tau \\
D^{n} & =\lambda \int_{0}^{t} E\left[\left\{\ln \left(\frac{1+U h(t+\Delta t-\tau)}{1+U h(t-\tau)}\right)\right\}^{n}\right] d \tau
\end{aligned}
$$

Proof. See the Appendix.

The above expressions further simplify for GBM and JD.

Example 2 For the Merton (1976) JD process, i.e., when $h(t)=1$, we get

$$
\begin{aligned}
C^{n} & =\lambda \Delta t E\left[\{\ln (1+U)\}^{n}\right] \\
D^{n} & =0
\end{aligned}
$$

Furthermore,

$$
\begin{aligned}
E\left[Z_{t}\right] & =\left(\left(\mu-\frac{\sigma^{2}}{2}\right)+\lambda E[\ln (1+U)]\right) \Delta t \\
\operatorname{Var}\left[Z_{t}\right] & =\left(\sigma^{2}+\lambda E\left[\{\ln (1+U)\}^{2}\right]\right) \Delta t \\
\text { Skewness } & =\frac{1}{\sqrt{\Delta t}}\left[\frac{\lambda E\left[\{\ln (1+U)\}^{3}\right]}{\left(\sigma^{2}+\lambda E\left[\{\ln (1+U)\}^{2}\right]\right)^{3 / 2}}\right] \\
\text { Kurtosis } & =3+\frac{1}{\Delta t}\left[\frac{\lambda E\left[\{\ln (1+U)\}^{4}\right]}{\left(\sigma^{2}+\lambda E\left[\{\ln (1+U)\}^{2}\right]\right)^{2}}\right] .
\end{aligned}
$$

See also Das and Sundaram (1999) and Aït-Sahalia $(2004)^{10}$.

To analyze the Shot-Noise effects on the higher-order moments of the SNJD model, it can be interesting to compare the skewness and excess kurtosis generated by the SNJD model under different degrees of autocorrelation. Tables $^{11} 3$ and 4 display the skewness and excess kurtosis coefficients (in rows) obtained from equations (11)-(12). Each Table contains four blocks of columns: the first one provides the parameters of the jump component of the

\footnotetext{
${ }^{10}$ Ait-Sahalia (2004) also provides an expression for the centered, absolute moments of non-integer order.

${ }^{11}$ These Tables are directly inspired by Das and Sundaram (1999).
} 
SNJD model. Jumps are normally distributed with $\ln (1+U) \sim N\left(\theta, \beta^{2}\right)$. Again, the SNJD model has been simulated by using an exponential response function. The second to fourth blocks correspond to the frequencies under study: daily, weekly and monthly, respectively. Each block contains the skewness and excess kurtosis for different values of $a(0.00,0.50,1.00$ and 10.00).

\section{[INSERT TABLES 3 AND 4 ABOUT HERE]}

By taking into account the expression for the total variance of the JD process $\sigma^{2} \Delta t+\lambda \Delta t E\left[\{\ln (1+U)\}^{2}\right]$ it is possible to provide an intuitive explanation of the results: For example, a parameter $\beta=0.02$ represents about $10 \%$ of contribution of the jumps to the total variance; similarly, values of $\beta=0.04,0.06$ constitute $36 \%$ and $64 \%$ over the total variance of the JD process owing to jumps.

One main conclusion arises from Tables 3 and 4: the serial dependence introduced by the SNJD model affects the results. By and large, it seems clear that the case $a=0.00$ exhibits different skewness and excess kurtosis coefficients from the remaining cases. These differences increase across the time intervals: the lower the frequency is, the higher the impact of the serial correlation in skewness and excess kurtosis is. For example, the differences in skewness between a JD process $(a=0.00)$ and the SNJD process with high persistence $(a=10.00)$ range from $10 \%$ for daily frequency to $60 \%$ for monthly frequency. When it comes to the excess kurtosis coefficients, these differences vary from $14 \%$ to $55 \%{ }^{12}$.

Some additional conclusions are obtained from Table 3. For example, the Shot-Noise effects in the SNJD model seem to be an additional source of asymmetries in returns. This is observable by modifying the parameter $\theta$, which controls for the jump mean. For example, no skewness is produced with symmetric jumps $(\theta=0.00)$ for $a=0.00$. By contrast, a positive skewness is observed in the remaining $a$ cases. Although this asymmetry effect is also noticed across frequencies, it seems to decrease with the frequency.

Similarly to the case of skewness, the Shot-Noise has effects on the excess kurtosis coefficients. For instance, the different values of excess kurtosis observed for the case without persistence $(a=0.00)$ range from $8 \%$ to $10 \%$ in daily frequencies.

\footnotetext{
${ }^{12}$ Das and Sundaram (1999) mention that the rate of decay of skewness and kurtosis in JD models is faster than that empirically observed. According to this, the authors notice that implied volatility smiles should not exist at long-periods (three months), as it is contrary to the market. This could be considered as a drawback of the JD models.
} 
Finally, Tables 3 and 4 show that, as the time-step decreases, the value of coefficients also decrease and tend, by the Central Limit Theorem, to those of a Normal distribution.

\subsection{Cross moments}

The cumulative Shot-Noise term $H_{t}$ (see 6 ) introduces some kind of serial dependence in log-returns, as could be seen in Table 2. This features should also be found in the cross moments of the series generated by the model (2).

The next Lemma provides the autocovariance for the log-returns in model (2).

Lemma 2 For $n \geq 1$, the autocovariance of the SNJD process is given by

$$
\begin{aligned}
& \operatorname{Cov}\left[Z_{t+n \Delta t}, Z_{t}\right]=\sigma^{2} \Delta t \delta(n)+\lambda E\left[U^{2}\right] \Delta t \delta(n) \\
& +\lambda \int_{0}^{t} E\left[\ln \left(\frac{1+U h(t+\Delta t-\tau)}{1+U h(t-\tau)}\right) \ln \left(\frac{1+U h(t+(n+1) \Delta t-\tau)}{1+U h(t+n \Delta t-\tau)}\right)\right] d \tau \\
& +\operatorname{Cov}\left[\sum_{j=N_{t}+1}^{N_{t+\Delta t}} U_{j}, \sum_{j=N_{t}+1}^{N_{t+n \Delta t}} U_{j}\left(h\left(t+n \Delta t-\tau_{j}\right)-h\left(t-\tau_{j}\right)\right)\right]
\end{aligned}
$$

where $n$ indicates the corresponding lag and $\delta(\cdot)$ denotes the Dirac delta function.

Proof. See the Appendix

Again, as expected, this result generalizes those obtained for GBM and JD processes since, in these cases, the right-hand side of (21) equals zero.

\section{THE SNJD MODEL WITH EXPONENTIAL DECAYING FUNCTION}

The SNJD model with exponential response function constitutes the simplest and most intuitive form of introducing a deterministic behavior after the jump into the model posited by Altmann et al. (2008). This section provides the main first moments and correlation expressions for this particular case of the SNJD model. 
By replacing the exponential response function in the moments derived in Lemma 1, we obtain

$$
\begin{aligned}
E\left[Z_{t}\right] & =\left(\mu-\frac{\sigma^{2}}{2}\right) \Delta t \\
\operatorname{Var}\left[Z_{t}\right] & =\left(\sigma^{2}+\lambda E\left[U^{2}\right]\right) \Delta t \\
\text { Skewness } & =\frac{1}{\sqrt{\Delta t}}\left[\frac{\lambda E\left[U^{3}\right]}{\left(\operatorname{Var}\left[Z_{t}\right]\right)^{3 / 2}}\right] \\
\text { Kurtosis } & =3+\frac{1}{\Delta t}\left[\frac{\lambda E\left[U^{4}\right]}{\left(\operatorname{Var}\left[Z_{t}\right]\right)^{2}}\right]
\end{aligned}
$$

Notice that expressions (22)-(25) reduce to those of the JD process in Example 2. Then, it seems that the SNJD process with exponential response function is similar to a JD process, at least in their first four moments. More precisely, the SNJD with exponential decaying function and the JD processes are almost equal in their first four moments. Notice that i) terms into expectations differ from $E\left[\ln (1+U)^{n}\right]$ for a JD process, with those of $E\left[U^{n}\right]$ for a SNJD process ${ }^{13}$ and ii) their main differences arise in their cross moments. To illustrate this last point, consider the following example where we provide an expression for the autocovariance of the SNJD process.

Example 3 For the exponential decay response function, the autocovariance for the log-returns of the process (2) is given by

$$
\begin{aligned}
\operatorname{Cov}\left[Z_{t+n \Delta t}, Z_{t}\right]= & {\left[\sigma^{2}+\lambda E\left[U^{2}\right]\right] \Delta t \delta(n) } \\
& +\lambda(\exp (-a \Delta t)-1) E\left[U^{2}\right] \Delta t \exp (-a n \Delta t)
\end{aligned}
$$

where $n$ indicates the corresponding lag and $\delta(\cdot)$ denotes the Dirac delta function.

Proof. See the Appendix

The first term in equation (26) reflects the contribution of the GBM and JD parts to the autocovariance of the SNJD process. As this expression reflects, this term is null for non-zero lags which means no additional contribution to the total variance of the process from the past. However, the

\footnotetext{
${ }^{13}$ Again, the origin of these differences is related to the numerical approximations involved in the computations of the SNJD process.
} 
last term in (26) shows that this covariance has an exponential decaying pattern owing to the Shot-Noise term. In other words, an additional source of covariance in the SNJD process is contained in past information.

Once the autocovariance of the SNJD process with the exponential response function (see equations (23)-(26)) has been obtained, the next Example provides an expression for the autocorrelation.

Example 4 For the exponential decay response function, the autocorrelation for the log-returns of the process (2) is given by

$$
\operatorname{corr}\left[Z_{t+n \Delta t}, Z_{t}\right]= \begin{cases}1 & \text { if } n=0 \\ (\exp (-a \Delta t)-1) \frac{\lambda E\left[U^{2}\right]}{\sigma^{2}+\lambda E\left[U^{2}\right]} \exp (-a n \Delta t) & \text { if } n \geq 1\end{cases}
$$

An inspection of this result reveals the following features:

- For positive values of $a$, the autocorrelation is always negative. As a function of this parameter, the autocorrelation attains its minimum at $a^{*}=\ln (n+1 / n) / \Delta t$, has an inflection point at $2 a^{*}$ and converges asymptotically to zero when $a$ tends to infinity. Figure 4 illustrates graphically these facts. Additionally, Figure 5 graphs the correlation as a function of the parameters $a$ and $n$.

- For $a=a^{*}$, the minimum autocorrelation, corr ${ }^{*}$, takes the value

$$
\operatorname{corr}^{*}=-\frac{\lambda E\left[U^{2}\right]}{\sigma^{2}+\lambda E\left[U^{2}\right]} \frac{n^{n}}{(n+1)^{n+1}}
$$

Thus, the minimum autocorrelation depends on the volatilities of the jump and Brownian motion components and the corresponding lag. Moreover, from (28), we obtain that the jump volatility is given as

$$
E\left[U^{2}\right]=-\frac{\sigma^{2}}{\lambda} \frac{\operatorname{corr}^{*}(n+1)^{n+1}}{n^{n}+\operatorname{corr}^{*}(n+1)^{n+1}}
$$

establishing a link between this volatility and the minimum autocorrelation of the log-returns for the exponential decay response function.

- The factor $\lambda E\left[U^{2}\right] /\left(\sigma^{2}+\lambda E\left[U^{2}\right]\right)$ represents the relative importance of the jump volatility with respect to that of the whole process. 


\section{[INSERT FIGURES 4 AND 5 ABOUT HERE]}

To illustrate numerically $(27)$, Table 5 includes the sample $\left(\widehat{\rho}_{n}\right)$ and theoretical autocorrelation values $\left(\rho_{n}^{\text {theo }}\right)$ of the SNJD model. The first column contains the number of lags. Each successive pair of columns exhibit, respectively, the theoretical ACF's obtained from equation (27) and the mean of a sample ACF coefficients of 100 simulation paths each with 1,800 steps. Finally, this experiment is repeated for different values of the parameter $a$.

\section{[INSERT TABLE 5 ABOUT HERE]}

Some conclusions arise from this Table:

1. As expected, the parameter $a$ controls the persistence of the process over time.

2. The theoretical autocorrelation values for the first few lags of $a=0.6$ and 1.0 seem to be closer to the sample autocorrelations than those obtained for $a=0.2$ and 10.0. This may be a consequence of the approximations carried out.

3. Any autocorrelation value for the SNJD process under study exhibits negative values, in contrast to the Shot-Noise results (see Table 1).

\section{ESTIMATION METHODOLOGY}

This section provides the econometric framework to estimate the parameters of the SNJD model. Our approach is based on the GMM estimate of Hansen (1982). The mathematical notation employed here has been taken from Hamilton (1994).

\subsection{General setting}

Once the moments of the SNJD model have been computed (see equations (22)-(25) and (27)), we employ the GMM to estimate the SNJD parameters. Basically, the main idea of this methodology consists of finding the parameters that match, as closely as possible, the population moments with their sample counterparts. This matching is obtained by minimizing the quadratic form

$$
J_{T}=\min _{\theta}[g(\theta)]^{\prime} W_{T}[g(\theta)]
$$


where $g(\theta)$ is a vector that contains the sample and population moments, and consequently, the unknown parameters vector $\theta$ that we are looking for. Additionally, $W_{T}$ is called the weighting matrix. The subindex $T$ denotes the sample size.

We consider three alternative weighting matrices: Firstly, to keep intuition in equation (30), $W_{T}$ will be equal to the Identity matrix. In this case, we are minimizing the sum of quadratic errors between sample and population moments. The estimate $J_{T}$ is known as the classical method of moments (Hamilton, 1994), it is consistent and asymptotically normal distributed and will be called first stage estimate (Cochrane, 2005).

Secondly, we use the weighting matrix proposed in Hansen (1982). This author considers $W_{T}=S_{T}^{-1}$, where $S_{T}$ is a variance-covariance matrix given by

$$
S_{T}=\frac{1}{T} \sum_{t=1}^{T}\left[h\left(\theta_{0}\right)\right]\left[h\left(\theta_{0}\right)\right]^{\prime}
$$

where $h(\cdot)$ indicates the vector of error terms and $\theta_{0}$ denotes the true value of the parameter vector. Now, the estimate $J_{T}$ obtained by employing equation (31) is a consistent, asymptotically normal, and asymptotically efficient estimate (Cochrane, 2005) of the parameter vector $(\theta)$. This will be our second stage estimate.

Finally, to take into account the possible existence of serial correlation, we will consider the Newey-West (1987) estimate as an alternative to $S_{T}$. This alternative will be our third stage estimate and is given as

$$
\widehat{S}_{T}=\widehat{\Gamma}_{0, T}+\sum_{v=1}^{q}\left[1-\left(\frac{v}{(q+1)}\right)\right]\left(\widehat{\Gamma}_{v, T}+\widehat{\Gamma}_{v, T}^{\prime}\right)
$$

where $q$ indicates a truncation parameter and

$$
\widehat{\Gamma}_{v, T}=\frac{1}{T} \sum_{t=v+1}^{T}\left[h\left(\widehat{\theta}, w_{t}\right)\right]\left[h\left(\widehat{\theta}, w_{t-v}\right)\right]^{\prime}
$$

\subsection{Results}

The moment conditions used in this Section comprise equations (22)-(25). Owing to the ability of the SNJD process to generate serial dependence, we also include the information contained in cross moments by means of the correlation function in equation (27).

Taking everything into account, the parameter vector of log-returns in (2) results $\Lambda=(\mu, \sigma, \lambda, \beta, a)^{\prime}$, where $\mu$ and $\sigma$ are the drift and the volatility of 
the Brownian motion, respectively; $\lambda$ is the intensity of the Poisson process; $\beta$ is related to the impact of the jump, and the parameter $a$ indicates the speed of the decaying effect after a jump.

To check the suitability of the GMM procedure, we have carried out a Monte Carlo experiment simulating 3,000 sample paths composed of 1,800 data each one. Table 6 reports the results. The two first columns of this Table display the SNJD parameters and their simulation values, respectively. Then, three vertical blocks contain the results for the alternative weighting matrices aforementioned ${ }^{14}$.

\section{[INSERT TABLE 6 ABOUT HERE]}

To keep the main results of these simulations manageable, each block reports the mean, the percentiles $[2.5 \%, 97.5 \%]$ and the root mean squared error (RMSE) of the obtained estimates. Additionally, to compare across samples for different experiments, Pearson's coefficient of variation (CV) is also included.

Some features derived from this Table can be highlighted. Firstly, under the different weighting matrices employed, estimates of the parameter $a$ seem to remain equal. However, the efficiency and precision of these estimates is different in each case, as is reflected in the CV and RMSE values (e.g., when $a=0.6,1.0)$. The worst performance in terms of RMSE is obtained for $a=0.2$, where the methodology seems to be slightly biased.

Secondly, as expected, the inclusion of the third stage estimate increases the efficiency of all the estimates, reflected in both the percentile interval and the CV coefficients.

Finally, for estimates of the parameter $\lambda$, it is remarkable that the more efficient the matrix we use, the higher is the bias obtained (see, for instance, when $a=1.0$ ). Moreover, this bias increases with the serial correlation. In contrast, the opposite behavior is observed for the parameters $\sigma$ and $\beta$ (e.g. $\sigma$ and $\beta$ estimates in the third stage column go, respectively, from 1.72 to 1.37 and 7.72 to 6.18 when we move from $a=0.20$ to $a=1.00$ ). This fact suggests a possible compensation effect between the components of the SNJD variance: Brownian noise and Shot-Noise term.

After discussing Table 6 briefly, it can be interesting to obtain a visual idea of these results. With this aim, Figures 6 and 7 display the sample

\footnotetext{
${ }^{14}$ For the sake of brevity, we have omitted the mean sample autocorrelation coefficients for all the experiments. On the whole, it can be said that these series exhibit significant sample autocorrelation values for one and two lags. This justifies the usage of the NeweyWest (1987) weighting matrix estimate. The results concerning these values are available upon request.
} 
distribution for first to third stage estimates of the parameters $\sigma, \lambda$ and $a$ in the case $a=0.6$. Graphs in columns are equally scaled for the horizontal axis.

\section{[INSERT FIGURES 6 AND 7 ABOUT HERE]}

As expected, these figures reveal that the third stage estimate seems to offer more efficient results than the others. The sample distributions obtained for the first and second stage estimates depict shapes which are different from the normal distribution, a fact that is owing to the presence of autocorrelation in the sample. Once again, the sample distributions obtained for the parameter $a$ present problems in the left tail (some observations (less than $5 \%$ of the total amount in the worst case) are around zero). Apparently, there is no bias in the obtained results.

\section{CONCLUSIONS}

To a certain extent, the formulation of modern finance from Black and Scholes (1973) to our days is based on continuous-time stochastic processes. Of all the available processes, the AJD model family studied by Duffie et al. (2000) seems to offer an optimum balance between empirical adequacy and analytical tractability. However, several recent empirical studies raise the question about considering new models to capture some of the drawbacks present in the existing models by, of course, keeping the usual requirements about parsimony in the number of variables and economic intuition.

This paper has presented a model that tries to fulfill the former requests. We focus on the SNJD model posited by Altmann et al. (2008). Basically, it is an extension of the JD models proposed by Merton (1976) where an additional term (named Shot-Noise function) is added to the Poisson process. The intuition behind this model is that a shock effect can fade away on the long run. As a result of this, the SNJD model can generate serial persistence in asset returns. As far as we know, this is the first study that analyzes in detail the main features of this model.

We have provided a general treatment of the Shot-Noise process in the economic literature. To the best of our knowledge, no similar analysis has been found in the financial literature. We have also provided an expression for log-returns of the SNJD model, identifying the source of autocovariance in the process. Not surprisingly, we find that the SNJD model is capable of nesting former versions of continuous-time processes such as GBM or JD ones. 
We have computed the characteristic function of the SNJD process, deriving some of its main moments and providing some useful numerical approximations for computational purposes. Regarding the dynamics of the model, we have analyzed the covariance function of the SNJD process. As we have pointed out, we observe that a considerable source of information is embedded in past information.

In addition to this, an extension to the paradigm of the SNJD models, the SNJD model with exponential decaying response function, has also been studied. Our results seem to conclude that the SNJD model with this response function is close to the JD version if we focus on their central moments, differing in their autocorrelation function specifications. It could be interesting to remark that we find a link between the serial correlation and the jump parameters.

Finally, we have also provided an estimation methodology for the SNJD model parameters based on the GMM estimate of Hansen (1982). With the purpose of checking the posited methodology, an exhaustive Monte Carlo study has been carried out. Our results reveal the presence of a certain bias for the parameter related to the jump probability. This bias may be explained in terms of a compensation effect between the components of the SNJD variance: Brownian noise and Shot-Noise term. In any case, we consider that the GMM framework is suitable for capturing the parameters of the SNJD model.

To conclude, for further research, we suggest studying the implications of the SNJD model from a financial point of view in depth. How deep the impact of the autocorrelation is on option prices or asset allocation strategies is a question which remains open. Moreover, the influence of Shot-Noise effects on, for example, electricity forward contracts is not clear. Taking these issues into account, this paper must be intended as a first attempt to analyze the main features of the SNJD model. 


\section{ACKNOWLEDGMENTS}

We are very grateful for the comments received from A. Cartea, A. León, M.A. Martínez, V. Meneu, A. Novales, D. Nualart, G. Rubio and from the attendants at the $63^{\text {rd }}$ Econometric Society European Meeting (ESEM-08), XIV Foro de Finanzas and IX Italian-Spanish Congress on Financial and Actuarial Mathematics, where a previous version of this paper received the Best Paper Award in the category "Investments and Financial Mathematics". Moreno gratefully acknowledges financial support by DGESIC grant SEJ2005-3924 and JCCM grant PCI08-0089-0766. 


\section{Appendix}

\section{Proof of Proposition 1}

The representation $Z_{t}=X_{t}+Y_{t}+H_{t}$ follows inmediately from (2) and

$$
\begin{aligned}
\frac{S_{t+\Delta t}}{S_{t}}= & \exp \left[\left(\mu-\frac{\sigma^{2}}{2}\right) \Delta t+\sigma\left(B_{t+\Delta t}-B_{t}\right)\right] \\
& \times \prod_{j=N_{t}+1}^{N_{t+\Delta t}}\left[1+U_{j} h\left(t+\Delta t-\tau_{j}\right)\right] \\
& \times \prod_{j=1}^{N_{t}} \frac{\left[1+U_{j} h\left(t+\Delta t-\tau_{j}\right)\right]}{\left[1+U_{j} h\left(t-\tau_{j}\right)\right]}
\end{aligned}
$$

That $X_{t}, Y_{t}$ and $H_{t}$ are independent follows from the independence of $B, N$ and the $U$ 's and the fact that $N$ has independent and stationary increments. To prove (7) recall the characteristic function of a normal variable to get the first factor. The arguments for the remaining two are similar. Therefore we only study $H_{t}$ in detail.

Now, given $N_{t}=m$, the jumps $\tau_{1}<\ldots<\tau_{m}$ have the same distribution as the order statistics of a sample of $m$ i.i.d. random variables uniformly distributed on $[0, t]$. See Parzen (1962). Thus,

$$
\begin{aligned}
& \Phi_{H_{t}}(\xi)=E\left[\exp \left(i \xi H_{t}\right)\right]= \\
= & E\left[\exp \left\{i \xi \sum_{j=1}^{N_{t}} \ln \left[\frac{1+U_{j} h\left(t+\Delta t-\tau_{j}\right)}{1+U_{j} h\left(t-\tau_{j}\right)}\right]\right\} \mid N_{t}=m\right] \times P\left(N_{t}=m\right) \\
= & \sum_{m=0}^{+\infty}\left[\frac{(\lambda t)^{m}}{m !} \exp (-\lambda t) \times \frac{m !}{(t)^{m}} \int_{0}^{t} d \tau_{1} \int_{\tau_{1}}^{t} d \tau_{2} \ldots \int_{\tau_{m-1}}^{t} d \tau_{m}\right] \\
& \times \prod_{j=1}^{m} E\left[\exp \left\{i \xi \ln \left(\frac{1+U_{j} h\left(t+\Delta t-\tau_{j}\right)}{1+U_{j} h\left(t-\tau_{j}\right)}\right)\right\}\right] \\
= & \exp (-\lambda t) \sum_{m=0}^{\infty}\left[\frac{(\lambda t)^{m}}{m !} \times\left[\frac{1}{t} \int_{0}^{t} E\left[\exp \left\{i \xi \ln \left(\frac{1+U h(t+\Delta t-\tau)}{1+U h(t-\tau)}\right)\right\}\right] d \tau\right]^{m}\right] \\
= & \exp \left\{\lambda \int_{0}^{t} E\left[\exp \left\{i \xi \ln \left(\frac{1+U h(t+\Delta t-\tau)}{1+U h(t-\tau)}\right)\right\}-1\right] d \tau\right\}
\end{aligned}
$$

This completes the proof of Proposition 1. 


\section{Proof of Lemma 1}

The non-central unconditional moments can be computed by differentiating expression (7), that is,

$$
\mu_{n}^{\prime}=E\left[Z_{t}^{n}\right]=\left.\frac{1}{i^{n}} \frac{\partial \Phi_{Z_{t}}}{\partial \xi}\right|_{\xi=0}
$$

In our case, the first four non-central moments are given as

$$
\begin{aligned}
\mu_{1}^{\prime}= & A+C+D \\
\mu_{2}^{\prime}= & \sigma^{2} \Delta t+C^{2}+D^{2}+\left(\mu_{1}^{\prime}\right)^{2} \\
\mu_{3}^{\prime}= & C^{3}+D^{3}-3(A+C+D)\left(2 B-C^{2}-D^{2}\right)+(A+C+D)^{3}(39) \\
\mu_{4}^{\prime}= & C^{4}+D^{4}+4(A+C+D)\left(C^{3}+D^{3}\right) \\
& +3\left(2 B-C^{2}-D^{2}\right)^{2}-5\left(2 B-C^{2}-D^{2}\right)(A+C+D) \\
& -\left(2 B-C^{2}-D^{2}\right)(A+C+D)^{2}+(A+C+D)^{4}
\end{aligned}
$$

with

$$
\begin{aligned}
A & =\left(\mu-\frac{\sigma^{2}}{2}\right) \Delta t \\
B & =-\frac{\sigma^{2}}{2} \Delta t \\
C^{n} & =\lambda \int_{t}^{t+\Delta t} E\left[\{\ln (1+U h(t+\Delta t-\tau))\}^{n}\right] d \tau \\
D^{n} & =\lambda \int_{0}^{t} E\left[\left\{\ln \left(\frac{1+U h(t+\Delta t-\tau)}{1+U h(t-\tau)}\right)\right\}^{n}\right] d \tau
\end{aligned}
$$

The proof is complete by applying the relationship between central and non-central moments (Abramowich and Stegun, 1968). 


\section{Proof of Lemma 2}

As the covariance is a linear operator and using the independence property between $B_{t}, N_{t}$ and $U_{j}$, we get

$$
\begin{aligned}
\operatorname{Cov}\left[Z_{t+n \Delta t}, Z_{t}\right] & =\operatorname{Cov}\left[X_{t+n \Delta t}, X_{t}\right]+\operatorname{Cov}\left[Y_{t+n \Delta t}, Y_{t}\right]+\operatorname{Cov}\left[H_{t+n \Delta t}, H_{t}\right] \\
& +\operatorname{Cov}\left[H_{t+n \Delta t}, Y_{t}\right], \quad n=0,1,2, \ldots
\end{aligned}
$$

The autocovariance of Brownian motion and Poisson process are easily obtained by applying standard arguments of independence between increments. We provide the results for the remaining cases:

1. Shot-Noise part. We get ${ }^{15}$

$$
\begin{aligned}
\operatorname{Cov}\left[H_{t+n \Delta t}, H_{t}\right] & =\lambda \int_{0}^{t} E\left[\ln \left(\frac{1+U h(t+\Delta t-\tau)}{1+U h(t-\tau)}\right)\right. \\
& \left.\times \ln \left(\frac{1+U h(t+\Delta t+n \Delta t-\tau)}{1+U h(t+n \Delta t-\tau)}\right)\right] d \tau(46)
\end{aligned}
$$

2. Jump / Shot-Noise part. The persistence in time of the realization of the random variable $U_{j}$ produces a cross effect between the processes $Y_{t}$ and $H_{t}$, which contributes to the appearance of a new source of autocovariance in the process $Z_{t}$.

$$
\begin{aligned}
& \operatorname{Cov}\left[Y_{t}, H_{t+n \Delta t}\right]= \\
&=\operatorname{Cov}\left[\sum_{j=N_{t}+1}^{N_{t+\Delta t}} U_{j}, \sum_{j=1}^{N_{t+n \Delta t}} U_{j}\left(h\left(t+n \Delta t-\tau_{j}\right)-h\left(t-\tau_{j}\right)\right)\right] \\
&=\operatorname{Cov}\left[\sum_{j=N_{t}+1}^{N_{t+\Delta t}} U_{j}, \sum_{j=N_{t}+1}^{N_{t+n \Delta t}} U_{j}\left(h\left(t+n \Delta t-\tau_{j}\right)-h\left(t-\tau_{j}\right)\right)\right]
\end{aligned}
$$

\footnotetext{
${ }^{15}$ For a formal proof of the covariance of the Shot noise, we refer the reader to Parzen (1962), Klüppelberg and Mikosch (1995) or Ross (1996).
} 


\section{Proof of Example 4}

As the Brownian and Jump parts of the autocovariance in the SNJD process are immediately computed, we focus on the Shot-Noise and Jump / Shot-Noise terms.

1. Shot-Noise part. Replacing $h(t)=\exp (-a t)$ into (46) and applying the power expansions of the logarithmic and exponential functions (around one and zero, respectively), we get

$$
\begin{aligned}
& \operatorname{Cov}\left[H_{t+n \Delta t}, H_{t}\right] \\
& =\lambda \int_{0}^{t} E\left[\ln \left(\frac{1+U \exp (-a(t+\Delta t-\tau))}{1+U \exp (-a(t-\tau))}\right)\right. \\
& \left.\quad \times \ln \left(\frac{1+U \exp (-a(t+(n+1) \Delta t-\tau))}{1+U \exp (-a(t+n \Delta t-\tau))}\right)\right] d \tau \\
& \simeq \quad \lambda E\left[U^{2}\right] \frac{(\exp (-a \Delta t)-1)^{2}}{2 a}(1-\exp (-2 a t)) \exp (-a n \Delta t) \\
& \simeq 0
\end{aligned}
$$

2. Jump / Shot-Noise part. Replacing $h(t)=\exp (-a t)$ into (47), applying the linearity of the covariance operator and the independence between Poisson increments, we obtain

$$
\begin{aligned}
\operatorname{Cov}\left[H_{t+n \Delta t}, Y_{t}\right] \\
=(\exp (-a \Delta t)-1) \operatorname{Cov}\left[\sum_{j=N_{t}+1}^{N_{t+\Delta t}} U_{j}, \sum_{j=N_{t}+1}^{N_{t+n \Delta t}} U_{j} \exp \left(-a\left(t+n \Delta t-\tau_{j}\right)\right]\right. \\
=(\exp (-a \Delta t)-1) \operatorname{Cov}\left[\sum_{j=N_{t}+1}^{N_{t+\Delta t}} U_{j}, \sum_{j=N_{t}+1}^{N_{t+\Delta t}} U_{j} \exp \left(-a\left(t+n \Delta t-\tau_{j}\right)\right)\right] \\
+(\exp (-a \Delta t)-1) \operatorname{Cov}\left[\sum_{j=N_{t}+1}^{N_{t+\Delta t}} U_{j}, \sum_{j=N_{t+\Delta t}+1}^{N_{t+n} \Delta t} U_{j} \exp \left(-a\left(t+n \Delta t-\tau_{j}\right)\right)\right] \\
=(\exp (-a \Delta t)-1) \operatorname{Var}\left(Y_{t}\right) \exp (-a n \Delta t)
\end{aligned}
$$




\section{References}

[1] Abramovich, M. and Stegun, I.A. (1968), Handbook of Mathematical Functions (3rd ed.), New York: Dover Publications.

[2] Aït-Sahalia, Y. (2004), "Disentangling Diffusion from Jumps," Journal of Financial Economics, 74, 487-528.

[3] Altmann, T., Schmidt, T. and Stute, W. (2008), "A Shot Noise Model for Financial Assets," International Journal of Theoretical and Applied Finance, 11, 1, 87-106.

[4] Andersen, T. G., Benzoni, L. and Lund, J. (2002), "An Empirical Investigation of Continuous-Time Equity Return Models," Journal of Finance, 47, 1239-1284.

[5] Ball, C.A. and Torous, W.N. (1983), "A Simplified Jump Process for Common Stock Returns," Journal of Financial and Quantitative Analysis, 18, 1, 53-65.

[6] (1985), "On Jumps in Common Stock Prices and Their Impact on Call Option Pricing," Journal of Finance, 40, 155-173.

[7] Barone-Adesi, G. and Gigli, A. (2003), "Managing Electricity Risk," Economic Notes, 32, 2, 283-294.

[8] Benito, F., León, A. and Nave, J. (2007), "Modeling the Euro Overnigth Rate," Journal of Empirical Finance, 14, 5, 756-782.

[9] Black, F. and Scholes, M. (1973), "The Pricing of Options and Corporate Liabilities," Journal of Political Economy, 81, 637-659.

[10] Bondesson, L. (1988), "Shot-Noise Processes and Distributions," Encyclopedia of Statistical Science, Vol. 8, New York: Wiley.

[11] Campbell, J.Y., Lo, A.W. and MacKinlay, A.C. (1996), The Econometrics of Financial Markets, Princeton, New Jersey: Princeton University Press.

[12] Cochrane, J. (2005), Asset Pricing, Princeton, New Jersey: Princeton University Press.

[13] Cox, J., Ingersoll, J. and Ross, S. (1985), "A Theory of the Term Structure of Interest Rates," Econometrica, 53, 2, 385-407. 
[14] Das, S. (2002), "The Surprise Element: Jumps in Interest Rates," Journal of Econometrics, 106, 27-65.

[15] Das, S. R. and Sundaram, R.K. (1999), "Of Smiles and Smirks: A Term Structure Perspective," Journal of Financial and Quantitative Analysis, 34, 2, 211-239.

[16] Duffee, G. (2002), "Term premia and Interest Rate Forecasts in Affine Models," Journal of Finance, 57, 1, 405-443.

[17] Duffie, D., Pan, J. and Singleton, K. (2000), "Transform Analysis and Asset Pricing for Affine Jump-Diffusions," Econometrica, 68, 13431376 .

[18] Duffie, D. and Gârleanu, N. (2001), "Risk and Valuation of Collateralized Debt Obligations," Financial Analysts Journal, January/February, 41-59.

[19] Escribano, A., Peña, J.I. and Villaplana, P. (2002), Modeling Electricity Prices: International Evidence, Working Paper, University Carlos III de Madrid, Department of Business Administration.

[20] Hamilton, J. (1994), Time Series Analysis, Princeton, New Jersey: Princeton University Press.

[21] Hansen, L. (1982), "Large Sample Properties of Generalized Method of Moments Estimators," Econometrica, 50, 1029-1054.

[22] Jorion, P. (1988), "On Jump Processes in the Foreign Exchange and Stock Markets," Review of Financial Studies, 1, 4, 427-445.

[23] Klüppelberg, C. and Mikosch, T. (1995), "Explosive Poisson Shot Noise Processes with Applications to Risk Reserves," Bernoulli, 1, 1/2, 125147.

[24] Lando, D. (2004), Credit Risk Modeling: Theory and Applications, Princeton, New Jersey: Princeton University Press.

[25] Lo, A. (1991), "Long-term Memory in Stock Market Prices," Econometrica, 59, 5, 1279-1313.

[26] Lucía, J. and Schwartz, E. (2001), "Electricity Prices and Power Derivatives: Evidence from Nordic Power Exchange," Review of Derivatives Research, 5, 1, 5-50. 
[27] Merton, R.C. (1976), "Option Pricing when Underlying Stock Returns are Discontinuous," Journal of Financial Economics, 3, 125-144.

[28] Navas, J. (2003), "Calculation of Volatility in a Jump-Diffusion Model," Journal of Derivatives, Winter, 66-72.

[29] Newey, W. and West, D. (1987), "A Simple Positive Semi-Definite, Heteroskedasticity and Autocorrelation Consistent Covariance Matrix," Econometrica, 55, 703-708.

[30] Parzen, E. (1962), Stochastic Processes, San Francisco: Holden Day Series in Probability and Statistics.

[31] Rice, S.O. (1954), "Mathematical Analysis of Random Noise," in Selected Papers on Noise and Stochastic Processes, ed. N. Wax, New York: Dover Publications.

[32] Ross, S.M. (1996), Stochastic Processes, John Wiley \& Sons, Inc.

[33] Schmidt, T. (2008), "Modelling Energy Markets with Extreme Spikes," in Mathematical Control Theory and Finance, eds. R. Grossinho, M. Guerra, A. Sarychev and A. Shiryaev. Springer.

[34] Singleton, K.J. (2001), "Estimation of Affine Asset Pricing Models Using the Empirical Characteristic Function," Journal of Econometrics, 102, 111-141.

[35] — (2006), Empirical Dynamic Asset Pricing, Princeton, New Jersey: Princeton University Press.

[36] Villaplana, P. (2003), Pricing Power Derivatives: a Two-Factor JumpDiffusion Approach, Working Paper, University Carlos III de Madrid, Department of Business Administration.

[37] Vasicek, O. (1977), "An Equilibrium Characterization of the Term Structure," Journal of Financial Economics, 5, 177-188.

[38] Weron, R. (2005), "Heavy Tails in Electricity Prices", Research Report HSC/05/2, Wroclaw University of Technology. 
Table 1: Autocorrelation function for different values of the parameter $a$.

\begin{tabular}{ccccc}
\hline Lag $(n)$ & $a=0.2$ & $a=0.6$ & $a=1.5$ & $A R(1), \phi=0.6$ \\
\hline & & & & \\
1 & 0.8187 & 0.5488 & 0.2231 & 0.6000 \\
2 & 0.6703 & 0.3012 & 0.0498 & 0.3600 \\
3 & 0.5488 & 0.1653 & 0.0111 & 0.2160 \\
4 & 0.4493 & 0.0907 & 0.0025 & 0.1296 \\
5 & 0.3679 & 0.0498 & 0.0006 & 0.0778 \\
10 & 0.1353 & 0.0025 & $3.06 \times 10^{-7}$ & 0.0060 \\
25 & 0.0067 & $3.06 \times 10^{-7}$ & $5.17 \times 10^{-17}$ & $2.84 \times 10^{-6}$ \\
50 & $4.54 \times 10^{-5}$ & $9.35 \times 10^{-14}$ & 0.0000 & $8.08 \times 10^{-12}$ \\
100 & $2.06 \times 10^{-9}$ & $8.75 \times 10^{-27}$ & 0.0000 & $6.53 \times 10^{-23}$ \\
& & & & \\
\hline
\end{tabular}

This Table reports the theoretical autocorrelation coefficients $\rho(n)$ for different lags and values of the parameter $a$ included in the response function $h\left(t-\tau_{k}\right)=\exp \left(-a\left(t-\tau_{k}\right)\right)$. Random variable $A_{k}$ is lognormally distributed as $A=\exp \left(-\beta^{2} / 2+\beta \varepsilon\right)-1$, with $\varepsilon \sim N(0,1)$. Simulation parameters are $\lambda=0.04$, $\beta=0.10$ and $a=0.50$. 
Table 2: Distributions and some statistics of GBM, JD and SNJD processes

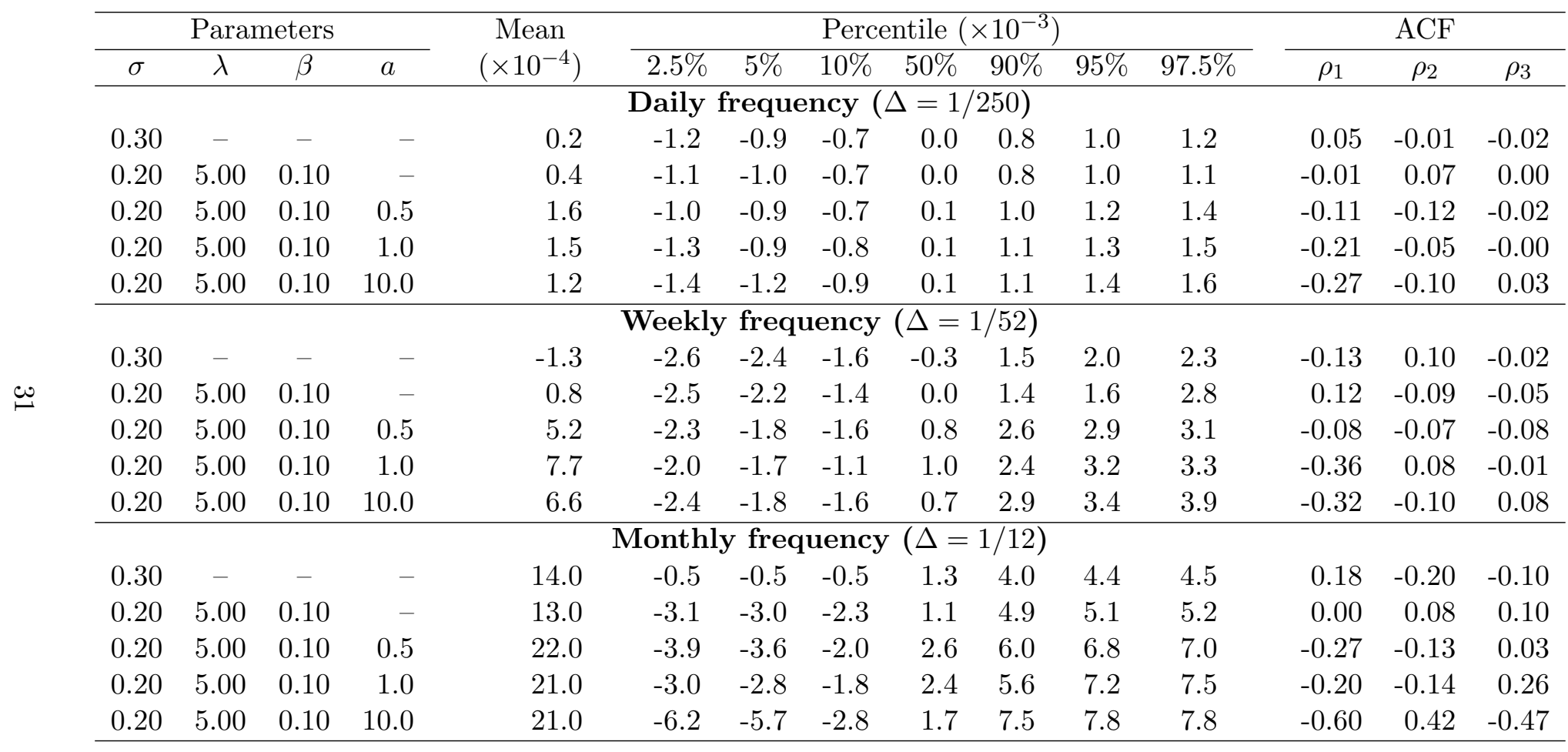

This Table includes the mean, some percentiles and the autocorrelation coefficients $\rho(n)$ for different lags and values for simulated samples of the GBM, JD and SNJD moels under different frequencies. Jump distribution in JD and SNJD models are lognormally distributed with $U=\exp \left(-\beta^{2} / 2+\beta \varepsilon\right)-1$, with $\varepsilon \sim N(0,1)$. The SNJD response function takes the form $h\left(t-\tau_{k}\right)=\exp \left(-a\left(t-\tau_{k}\right)\right)$. Parameter $\mu$ has been fixed to 0.20 in all cases. 
Table 3: Skewness in the SNJD process

\begin{tabular}{|c|c|c|c|c|c|c|c|c|c|c|c|c|c|c|}
\hline \multicolumn{3}{|c|}{ Parameters } & \multicolumn{4}{|c|}{$a(\Delta=1 / 250)$} & \multicolumn{4}{|c|}{$a(\Delta=1 / 52)$} & \multicolumn{4}{|c|}{$a(\Delta=1 / 12)$} \\
\hline$\sigma$ & $\theta$ & $\beta$ & 0.0 & 0.5 & 1.0 & 10.0 & 0.0 & 0.5 & 1.0 & 10.0 & 0.0 & 0.5 & 1.0 & 10.0 \\
\hline 0.24 & 0.00 & 0.02 & 0.000 & 0.008 & 0.007 & 0.007 & 0.000 & 0.003 & 0.003 & 0.003 & 0.000 & 0.002 & 0.001 & 0.000 \\
\hline 0.24 & -0.001 & 0.02 & -0.013 & -0.005 & -0.005 & -0.005 & -0.006 & -0.002 & -0.002 & -0.002 & -0.003 & -0.001 & -0.001 & -0.000 \\
\hline 0.24 & -0.100 & 0.02 & -2.737 & -2.562 & -2.557 & -2.464 & -1.243 & -1.155 & -1.143 & -0.954 & -0.597 & -0.537 & -0.514 & -0.230 \\
\hline 0.20 & 0.00 & 0.04 & 0.000 & 0.138 & 0.138 & 0.132 & 0.000 & 0.062 & 0.061 & 0.050 & 0.000 & 0.029 & 0.027 & 0.010 \\
\hline 0.20 & -0.001 & 0.04 & -0.058 & 0.080 & 0.080 & 0.077 & -0.026 & 0.036 & 0.036 & 0.029 & -0.013 & 0.017 & 0.016 & 0.006 \\
\hline 0.20 & -0.100 & 0.04 & -3.813 & -3.567 & -3.560 & -3.440 & -1.732 & -1.608 & -1.594 & -1.347 & -0.832 & -0.741 & -0.720 & -0.341 \\
\hline 0.15 & 0.00 & 0.06 & 0.000 & 0.655 & 0.654 & 0.630 & 0.000 & 0.295 & 0.292 & 0.244 & 0.000 & 0.137 & 0.131 & 0.059 \\
\hline 0.15 & -0.001 & 0.06 & -0.121 & 0.534 & 0.533 & 0.513 & -0.055 & 0.241 & 0.238 & 0.199 & -0.026 & 0.112 & 0.107 & 0.048 \\
\hline 0.15 & -0.100 & 0.06 & -5.233 & -4.923 & -4.915 & -4.764 & -2.377 & -2.222 & -2.203 & -1.892 & -1.142 & -1.038 & -1.001 & -0.509 \\
\hline
\end{tabular}

Skewness for the SNJD process. Jump size in returns is normally distributed with $\ln (1+U) \sim N\left(\theta, \beta^{2}\right)$. Response function in the SNJD process is of exponential decay type. The annual volatility has been fixed to $\sigma_{\text {annual }}=0.25$ for all cases. Parameter $\lambda$ (the average number of jumps per year) is also constant and equal to 10 . 
Table 4: Excess Kurtosis in the SNJD process

\begin{tabular}{|c|c|c|c|c|c|c|c|c|c|c|c|c|c|c|}
\hline \multicolumn{3}{|c|}{ Parameters } & \multicolumn{4}{|c|}{$\mathrm{a}(\Delta=1 / 250)$} & \multicolumn{4}{|c|}{$\mathrm{a}(\Delta=1 / 52)$} & \multicolumn{4}{|c|}{$\mathrm{a}(\Delta=1 / 12)$} \\
\hline$\sigma$ & $\theta$ & $\beta$ & 0.0 & 0.5 & 1.0 & 10.0 & 0.0 & 0.5 & 1.0 & 10.0 & 0.0 & 0.5 & 1.0 & 10.0 \\
\hline 0.24 & 0.00 & 0.02 & 0.319 & 0.319 & 0.318 & 0.297 & 0.066 & 0.065 & 0.064 & 0.047 & 0.015 & 0.014 & 0.013 & 0.005 \\
\hline 0.24 & -0.001 & 0.02 & 0.320 & 0.317 & 0.316 & 0.295 & 0.066 & 0.065 & 0.063 & 0.047 & 0.015 & 0.014 & 0.013 & 0.005 \\
\hline 0.24 & -0.100 & 0.02 & 12.012 & 10.933 & 10.903 & 10.387 & 2.479 & 2.232 & 2.203 & 1.774 & 0.572 & 0.493 & 0.467 & 0.260 \\
\hline 0.20 & 0.00 & 0.04 & 6.171 & 6.248 & 6.227 & 5.862 & 1.274 & 1.273 & 1.252 & 0.953 & 0.294 & 0.278 & 0.260 & 0.116 \\
\hline 0.20 & -0.001 & 0.04 & 6.177 & 6.202 & 6.181 & 5.819 & 1.274 & 1.263 & 1.243 & 0.946 & 0.294 & 0.276 & 0.258 & 0.115 \\
\hline 0.20 & -0.100 & 0.04 & 21.091 & 18.971 & 18.923 & 18.093 & 4.352 & 3.877 & 3.830 & 3.137 & 1.004 & 0.860 & 0.817 & 0.490 \\
\hline 0.15 & 0.00 & 0.06 & 28.630 & 29.449 & 29.368 & 27.977 & 5.908 & 6.013 & 5.935 & 4.784 & 1.363 & 1.329 & 1.258 & 0.700 \\
\hline 0.15 & -0.001 & 0.06 & 28.636 & 29.274 & 29.194 & 27.811 & 5.910 & 5.976 & 5.899 & 4.746 & 1.363 & 1.321 & 1.251 & 0.686 \\
\hline 0.15 & -0.100 & 0.06 & 35.598 & 31.993 & 31.920 & 30.651 & 7.346 & 6.544 & 6.472 & 5.413 & 1.695 & 1.456 & 1.392 & 0.916 \\
\hline
\end{tabular}

Skewness for the SNJD process. Jump size in returns is normally distributed with $\ln (1+U) \sim N\left(\theta, \beta^{2}\right)$. Response function in the SNJD process is of exponential decay type. The annual volatility has been fixed to $\sigma_{\text {annual }}=0.25$ for all cases. Parameter $\lambda$ (the average number of jumps per year) is also constant and equal to 10 . 
Table 5: Autocorrelation function for different values of the parameter $a$

\begin{tabular}{|c|c|c|c|c|c|c|c|c|}
\hline & \multicolumn{2}{|c|}{$a=0.2$} & \multicolumn{2}{|c|}{$a=0.6$} & \multicolumn{2}{|c|}{$a=1.0$} & \multicolumn{2}{|c|}{$a=10.0$} \\
\hline Lag & $\rho_{n}^{\text {theo }}$ & $\widehat{\rho}_{n}$ & $\rho_{n}^{\text {theo }}$ & $\widehat{\rho}_{n}$ & $\rho_{n}^{\text {theo }}$ & $\widehat{\rho}_{n}$ & $\rho_{n}^{\text {theo }}$ & $\widehat{\rho}_{n}$ \\
\hline 1 & -0.144 & -0.068 & -0.240 & -0.172 & -0.225 & -0.250 & 0.000 & -0.416 \\
\hline 2 & -0.118 & -0.057 & -0.132 & -0.096 & -0.083 & -0.091 & 0.000 & 0.001 \\
\hline 3 & -0.096 & -0.046 & -0.072 & -0.047 & -0.031 & -0.031 & 0.000 & -0.000 \\
\hline 4 & -0.079 & -0.038 & -0.040 & -0.029 & -0.011 & -0.013 & 0.000 & -0.005 \\
\hline 5 & -0.065 & -0.027 & -0.022 & -0.016 & -0.004 & -0.003 & 0.000 & 0.005 \\
\hline 10 & -0.024 & -0.013 & -0.001 & 0.005 & 0.000 & -0.001 & 0.000 & 0.002 \\
\hline 25 & -0.001 & 0.001 & 0.000 & -0.001 & 0.000 & 0.005 & 0.000 & -0.003 \\
\hline 50 & 0.000 & 0.001 & 0.000 & -0.001 & 0.000 & -0.001 & 0.000 & -0.001 \\
\hline 100 & 0.000 & -0.003 & 0.000 & -0.001 & 0.000 & -0.001 & 0.000 & -0.003 \\
\hline
\end{tabular}

This Table includes the sample, $\widehat{\rho}_{n}$, and theoretical, $\rho_{n}^{\text {theo }}$ (see equation (27)), autocorrelation values of the SNJD model for different values of the parameter $a$. Sample autocorrelation coefficients have been computed from 100 simulation paths with 1,800 steps of the SNJD model with response function $h\left(t-\tau_{k}\right)=\exp \left(-a\left(t-\tau_{k}\right)\right)$. The random variable $U$ is lognormally distributed as $U=\exp \left(-\beta^{2} / 2+\beta \varepsilon\right)-1$, with $\varepsilon \sim N(0,1)$. Simulated (annualized) parameters are $\mu=0.05, \sigma=0.20, \lambda=10.00, \beta=0.10$ and $\Delta t=1 / 250$. 
Table 6: GMM estimates for the SNJD process

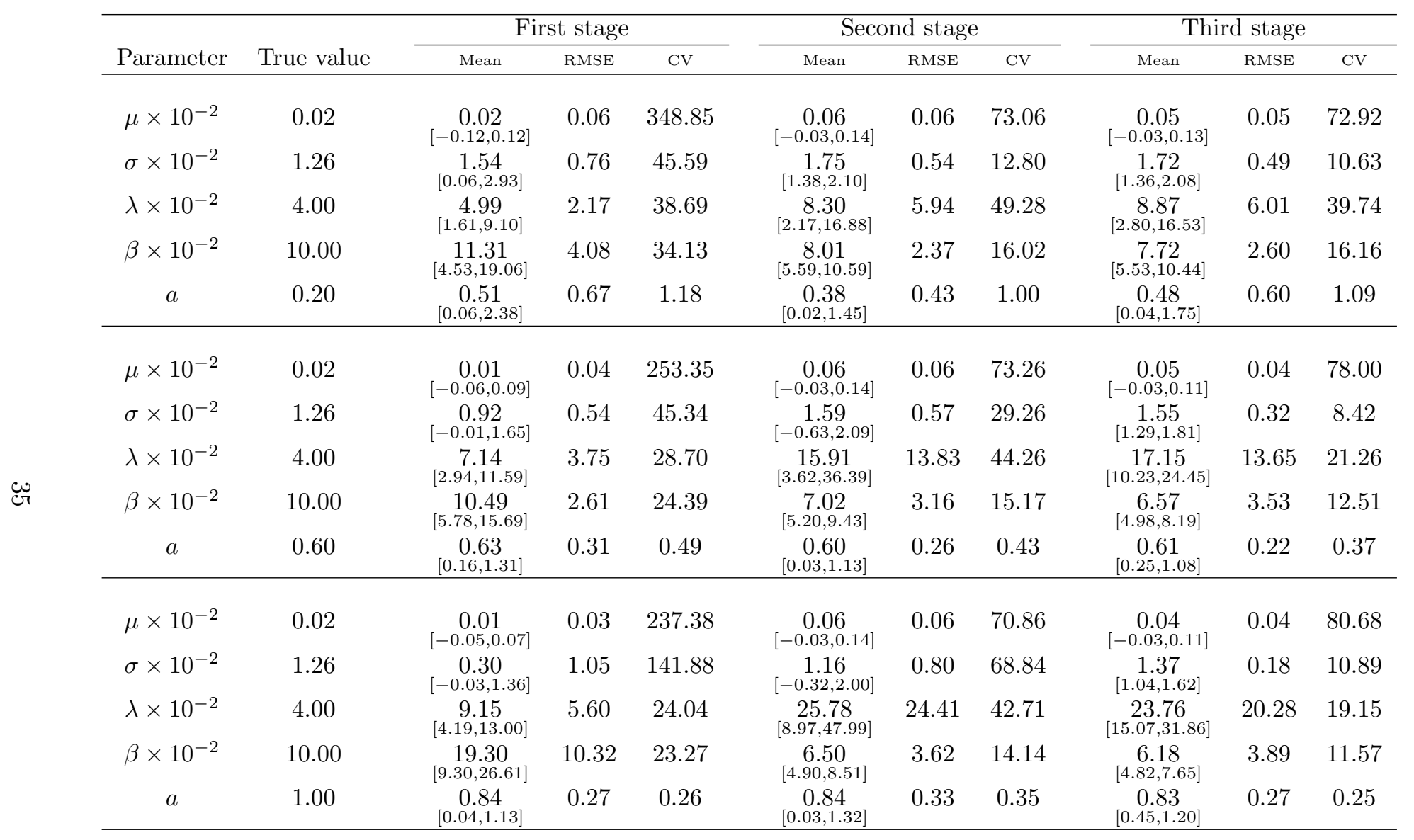

This Table includes the GMM (first, second and third stage) estimates of the SNJD process for different values of the parameter $a$. The first stage estimate corresponds to a GMM with the identity as weighting matrix. The second stages estimate uses the efficient version of the weighting matrix of Hansen (1982). Due to the presence of autocorrelation in the sample, we use the weighting matrix version of Newey-West (1987) as third stage estimate. We assume a jump size $U$ lognormally distributed of the form $U=\exp \left(-\beta^{2} / 2+\beta \varepsilon\right)-1$, with $\varepsilon \sim N(0,1)$, and response function of the form $h(t)=\exp (-a t)$. Each row refers the true simulation values, mean, RMSE and CV of first to third stage estimates for each parameter of the SNJD process, respectively. 


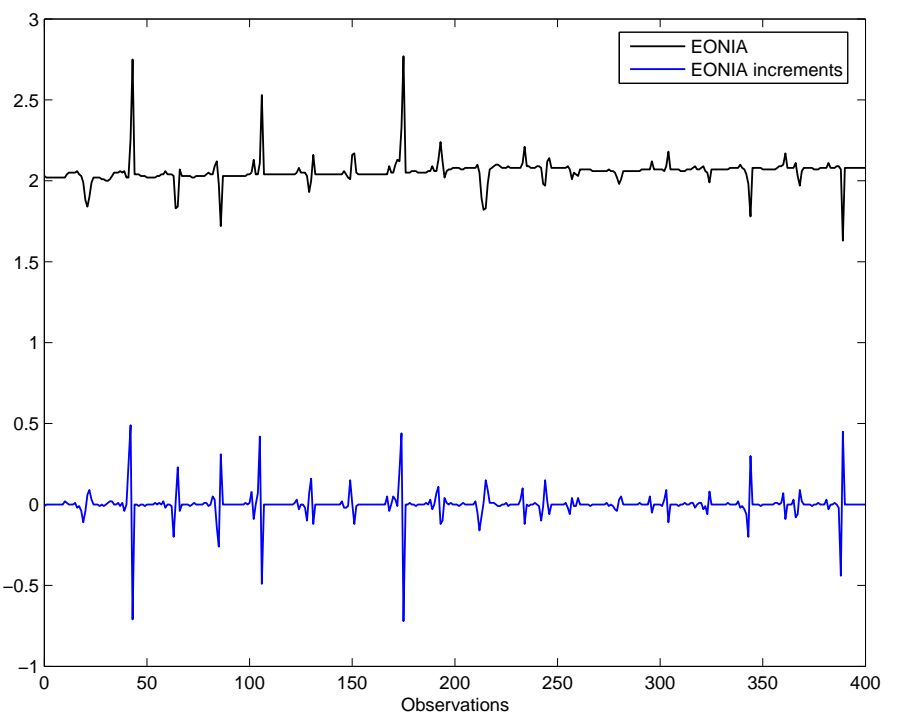

Figure 1: Sample path for the EONIA rate. Upper and lower lines display the sample path for levels and increments of this variable, respectively. 


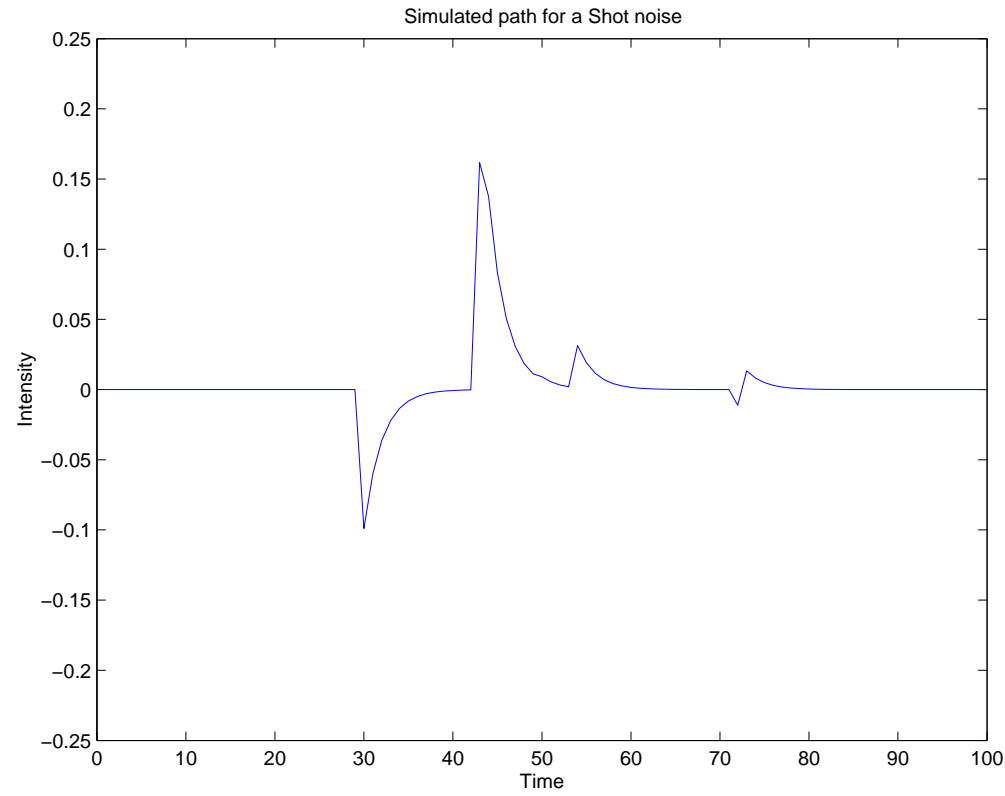

Figure 2: A simulated path of the Shot Noise process as given in expression (1). The response function is $h\left(t-\tau_{k}\right)=\exp \left(-a\left(t-\tau_{k}\right)\right)$. The random variables $A_{k}$ are lognormally distributed as $A=\exp \left(-\beta^{2} / 2+\beta \varepsilon\right)-1$, with $\varepsilon \sim N(0,1)$ and $\beta$ is a constant. Simulation parameters are $\lambda=10.00, \beta=0.10$ and $a=0.50$. 


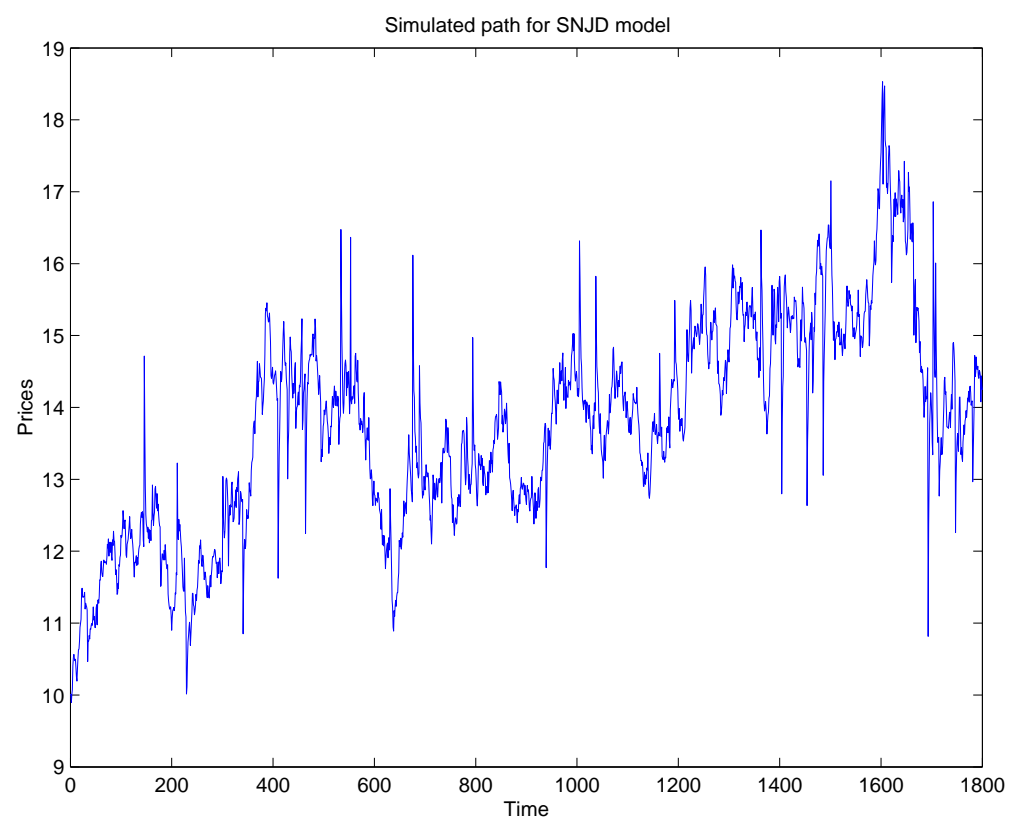

Figure 3: Representation of a simulated path of a SNJD (Altmann et al., 2008) process. The random variable $U$ is lognormally distributed as $U=\exp \left(-\beta^{2} / 2+\right.$ $\beta \varepsilon)-1$, with $\varepsilon \sim N(0,1)$. The (annualized) parameters are $S_{0}=10.00, \mu=0.05$, $\sigma=0.20, \lambda=10.00, \beta=0.05$ and $a=0.50$ 


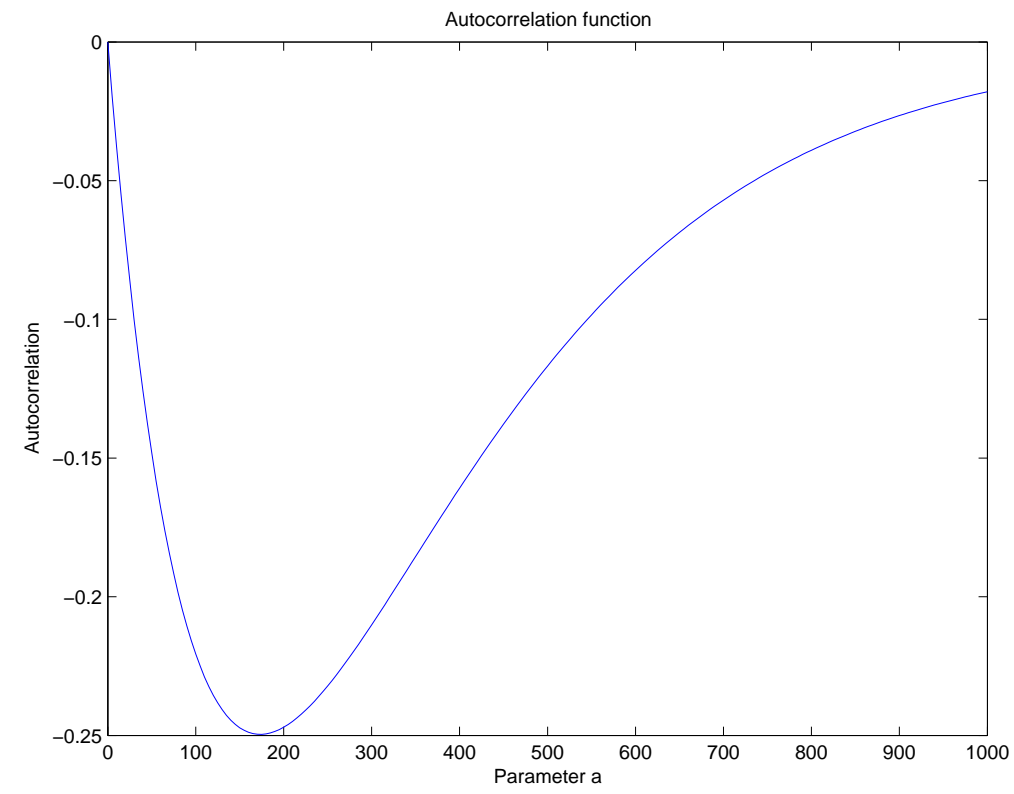

Figure 4: Autocorrelation function for the log-returns of the exponential decay function $h(t)=\exp (-a t)$ as a function of the parameter $a$. The random variable $U$ is lognormally distributed as $U=\exp \left(-\beta^{2} / 2+\beta \varepsilon\right)-1$, with $\varepsilon \sim N(0,1)$. Simulation parameters are $\sigma=0.20, \lambda=10.00, \beta=0.10, n=1$ and $\Delta t=1 / 250$. 


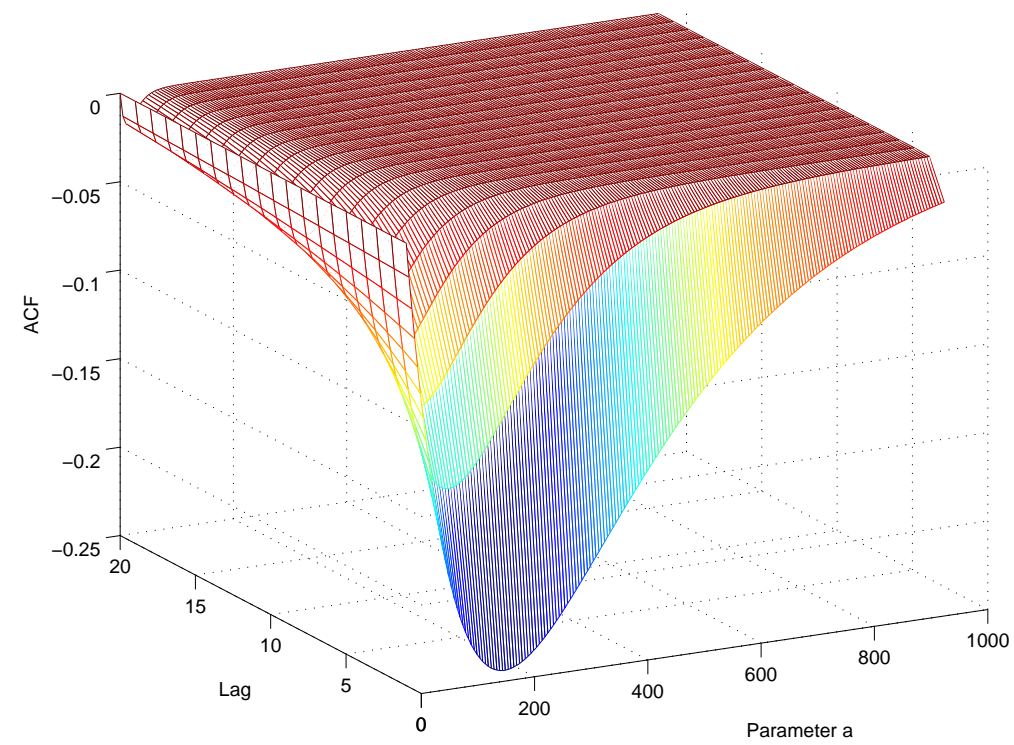

Figure 5: Autocorrelation function for the log-returns of the exponential decay function $h(t)=\exp (-a t)$ as a function of the parameters $a$ and $n$. The random variable $U$ is lognormally distributed as $U=\exp \left(-\beta^{2} / 2+\beta \varepsilon\right)-1$, with $\varepsilon \sim N(0,1)$. Simulation parameters are $\sigma=0.20, \lambda=10.00, \beta=0.10$ and $\Delta t=1 / 250$. 
Identity matrix (first stage estimate)
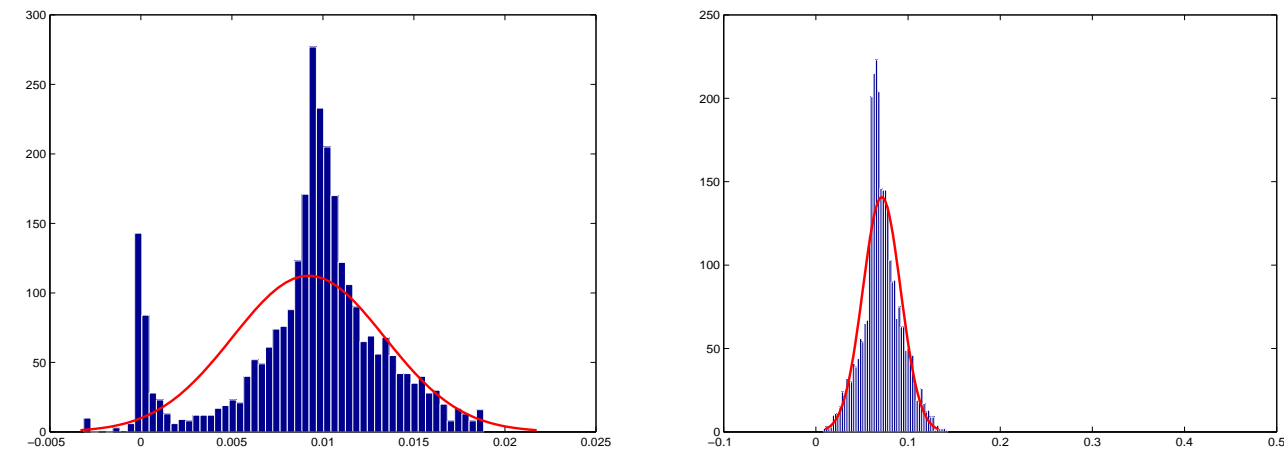

Hansen (1982) (second stage estimate)
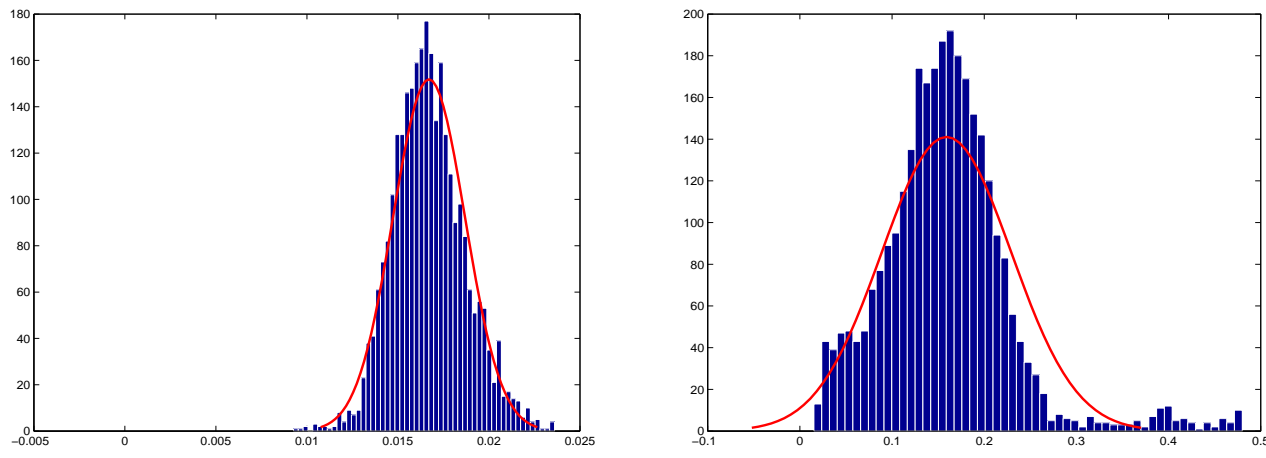

Newey-West (1987) (third stage estimate)
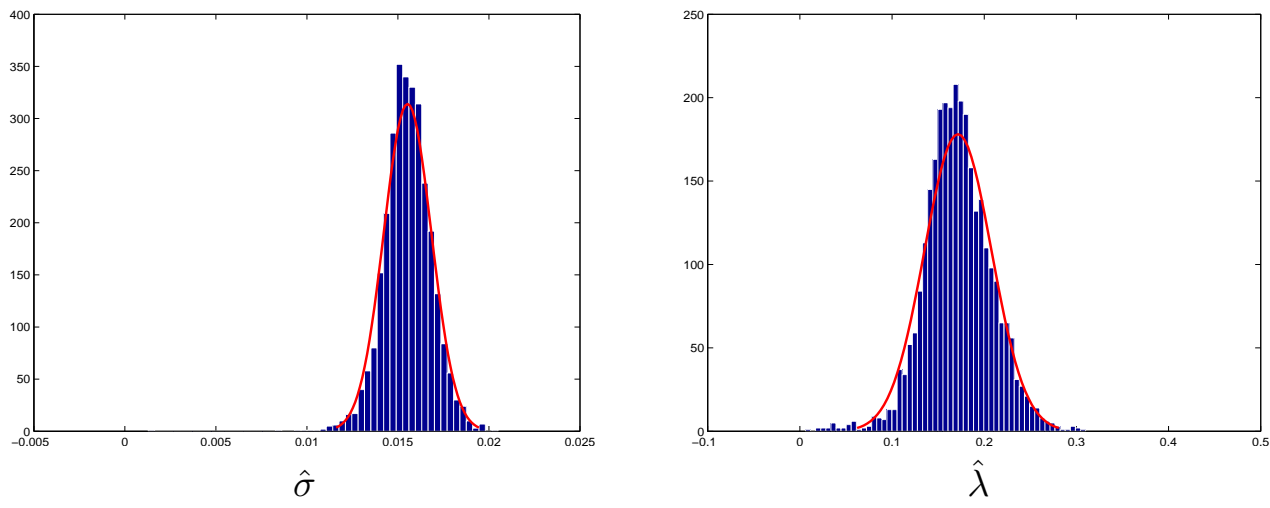

Figure 6: Sample distribution of estimated $\sigma$ and $\lambda$ SNJD parameters for different weighting matrices. Simulation values are $\mu=0.02 \times 10^{-2}, \sigma=1.26 \times 10^{-2}$, $\lambda=4.00 \times 10^{-2}, \beta=10.00 \times 10^{-2}$ and $a=0.60$. The random variable $U$ is lognormally distributed. Note that the axis OX are equally scaled. 
Identity matrix (first stage estimate)

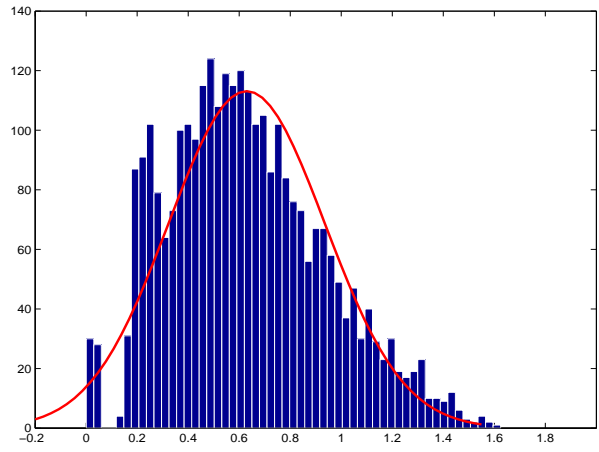

Hansen (1982) (second stage estimate)

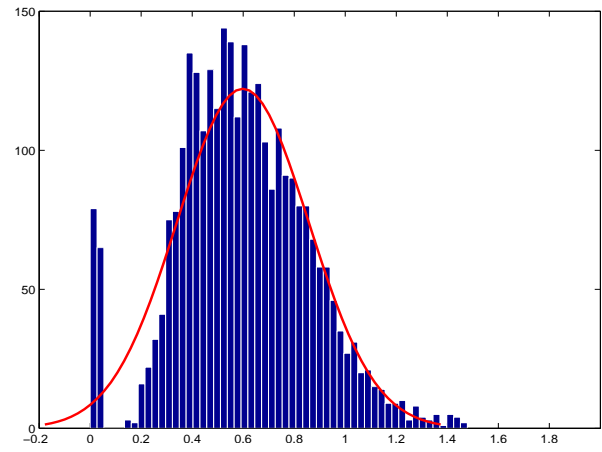

Newey-West (1987) (third stage estimate)

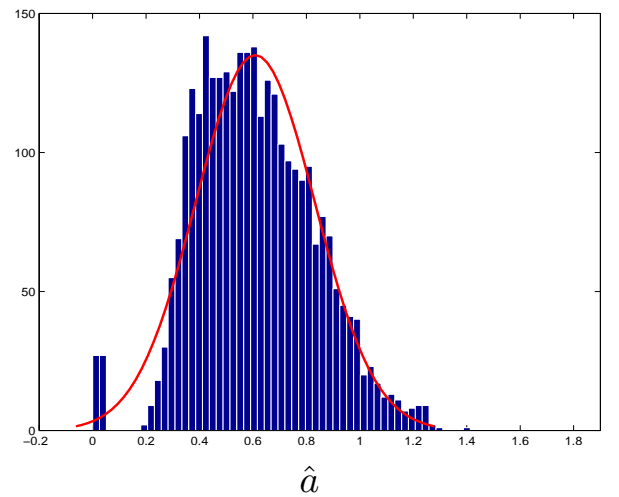

Figure 7: Sample distribution of estimated a SNJD parameter for different weighting matrices. Simulation values are $\mu=0.02 \times 10^{-2}, \sigma=1.26 \times 10^{-2}$, $\lambda=4.00 \times 10^{-2}, \beta=10.00 \times 10^{-2}$ and $a=0.60$. The random variable $U$ is lognormally distributed. Note that the axes OX are equally scaled in all the graphs. 